\title{
The Impact of Built Environment on Diabetic Patients: The Case of Eastern Province, Kingdom of Saudi Arabia
}

\author{
Bhzad Sidawi $^{1} \&$ Mohamed Taha Ali Al-Hariri ${ }^{2}$ \\ ${ }^{1}$ Department of Architecture, College of Architecture and Planning, Saudi Arabia \\ ${ }^{2}$ Department of Physiology, College of Medicine, University of Dammam, Dammam, Kingdom of Saudi Arabia \\ Correspondence: Bhzad Sidawi, Associate professor, Department of Architecture, College of Architecture and \\ Planning, Saudi Arabia
}

Received: May 21, $2012 \quad$ Accepted: June 4, $2012 \quad$ Online Published: June 13, 2012
doi:10.5539/gjhs.v4n4p126 $\quad$ URL: http://dx.doi.org/10.5539/gjhs.v4n4p126

\begin{abstract}
At present, Diabetes mellitus is considered as one of the main threats to the human health in the 21 st century. It may lead to severe conditions such as blindness, end-stage of renal disease, limb amputation and a variety of debilitating neuropathies. Previous researches indicated that diabetes is caused by a complex interaction of patient's genetics, life-style and environmental factors. They also highlighted that providing quality and healthy built environment to citizens including diabetic patients would prevent poor and unhealthy condition. The Kingdom of Saudi Arabia (KSA) is one of top ten countries in the prevalence of diabetes. Little researches though were conducted in KSA in regards to the effect of environmental conditions of the built environment. In 2011, the present researchers have carried out a pilot survey on a number of diabetic patients to find out the possible impact of built environment settings on the patient's lifestyle. The research explored whether diabetic patients use smart tools in their daily life to overcome the daily life's difficulties and perform their life as normal as possible. The results showed a close link between a poor home and environmental settings, the patient's lifestyle, and the patient's health status. It also highlighted the absence of smart tools and systems use. The paper argues that certain changes to the built environment must be done and to provide a healthy and safe environment for diabetic patients. This would help these patients to abandon their bad habits and adopt healthier lifestyle.
\end{abstract}

Keywords: diabetes, urban pollution, lifestyle, sick building syndrome, smart tools, built environment

\section{Introduction}

Diabetes is one of the most serious common non-communicable diseases that faces people worldwide and diabetic people are patients whose life relies on continuous support, care and monitoring. Researchers highlighted that diabetes is caused by a complex interaction of patient's genetics, life-style and environmental factors (see for instance Stewart et al., 2011). The bio-psychosocial model as a new paradigm recognizes that disease and behavior are functions and result from the interaction among biological, psychosocial, developmental, socio-cultural and ecological factors (Madhu \& Sridhar, 2001). Urban sprawl has been linked to a variety of health-related concerns including air pollution, water quality, traffic accidents, and mental health issues (Bray, Vakil, \& Elliott, 2005). The study indicates that urban design settings and various aspects of the built environment can also play an important role in the development of diabetes T2DM and its risk factors (Allanah, Ashley, \& Farley, 2010). The sick built environment may also enforce people to adopt habits and lifestyle that is unhealthy and complicate their health status. Studies of the built environment acknowledge that aspects of our physical surroundings can shape choices about diet and physical activity - both important contributors to the development of diabetes (ibid).

So the built environment should be properly designed to create a healthy environment and lifestyle. This includes the study of physical surroundings' features such as proximity of grocery stores, safe and pleasant opportunities for physical activity, and time spent commuting. Changes should be made to the built environment to positively influence health outcomes and to be made more activity-friendly by improving sidewalks and bicycle paths, building recreation spaces, and instituting mixed land-use patterns in more suburban areas to provide better walking destinations (ibid). Smart tools would help people and especially with some type of disability to overcome the life difficulties and facilitate their access to places within the built environment. This paper focuses 
on the built environment and lifestyle factors that would contribute to the development of diabetes and discusses possible solutions that improve the health status of diabetic patients.

\section{The Diabetes Types and Complications}

Diabetes mellitus (DM) is defined by American Diabetic Association (ADA) and adopted by World Health Organization (WHO) as the following: an individual is said to have normal blood glucose when fasting plasma glucose (FPG) is $<6.1 \mathrm{mmol} / \mathrm{L}(110 \mathrm{mg} / \mathrm{dL})$, impaired fasting glucose (IFG) when FPG is between 6.1-6.9 $\mathrm{mmol} / \mathrm{L}(110$ and $125 \mathrm{mg} / \mathrm{dL})$, and DM when $\mathrm{FPG}$ is $\geq 7.0 \mathrm{mmol} / \mathrm{L}(126 \mathrm{mg} / \mathrm{dL})$ or a random value at or above $11.1 \mathrm{mmol} / \mathrm{L}(200 \mathrm{mg} / \mathrm{dL})$ (Alberti \& Zimmet, 1998). There are - in general- two types of diabetes. In Diabetes type I (T1DM), the person's own body has destroyed the insulin-producing beta cells in the pancreas. Although type II diabetes mellitus (T2DM) can be caused by genetic factors, unhealthy lifestyle happens to be the main cause. A person with T2DM has one of two problems, and sometimes both: a) not enough insulin is being produced; and b) the insulin is not working properly. Diabetes mellitus is the most common non-communicable disease worldwide and the fourth to fifth leading cause of death in developed countries. The International Diabetes Federation (IDF) indicates that the prevalence of diabetes mellitus has reached epidemic levels globally. Estimates for 2010 indicate that 285 million adults have diabetes in the seven regions of the IDF. These numbers represent an increase of 39 million from 2007 and an expected continued increase to 439 million in 2030 (International Diabetes Federation, 2009). While the direct symptoms of diabetes, such as thirst, frequent urination and fatigue, can be mild and may cause little interruption to activities of daily living, it is the complications of the disease, including blindness in adults (Jeppesen \& Bek, 2004) non-traumatic lower-limb amputation (Chaturvedi, Stevens, Fuller, Lee, \& Lu, 2001) and kidney failure resulting in transplantation and dialysis (Atkins, 2005). In addition, the risk of coronary heart disease is two to four times higher in diabetic patients. The risk of stroke or peripheral vascular disease also increases strongly. In fact, the management and treatment of diabetes mellitus mainly T2DM is can considered more than the mere control of blood glucose values, asking for a multidisciplinary approach (i.e. shared care) to reduce macro- and micro-vascular risk factors (American Diabetes Association, 2006).

\subsection{Diabetes and Socio-economic Factors}

Diabetes mainly T2DM is a lifestyle disorder, and many studies indicates that, the incidence of this disease is projected to increase as populations age (International Diabetes Federation, 2007), urbanization increases (Bray, Vakil, \& Elliott, 2005), diets become 'westernized' (Schulze, Manson, Willett, \& Hu, 2003), and levels of physical activity decrease (Eckel et al., 2004). Passive entertainment exemplified by television viewing and computer games along with intake of meals; all contribute to disorders of lifestyle (Peckhan, 1998, Ebbeling, Pawlak, \& Ludwig, 2002). This imbalance between energy intake (i.e. feeding) and energy expenditure (i.e. physical activity) unfortunately leads to obesity (Michaud et al., 2001, Ebbeling, Pawlak, \& Ludwig, 2002). Moreover, a growing body of evidence suggests an association between diabetes with socioeconomic and built environment conditions (Stewart et al., 2011).

Previous surveys from KSA suggested that diabetes is present in epidemic proportions throughout the country with exceedingly high rates concentrated in urban areas (Alzaid, 1997). A Study showed that, prevalence of diabetes mellitus is highest among the Northern Saudi population (Al-Nozha, Al-Maatouq, \& Al-Mazrou, 2004). A US study showed that, incidence of diabetes was higher among African-American women (i.e. black women) in low socioeconomic status (SES) versus higher SES neighborhoods (Krishnan, Cozier, Rosenberg, \& Palmer, 2010), greater risk of coronary heart disease in socioeconomically disadvantaged versus more affluent census block groups (Diex Roux et al., 2001), and higher rates of obesity in socioeconomically deprived neighborhoods compared to more affluent neighborhoods (Cubbin et al., 2006). Other investigations indicate higher rates of diabetes (Nemmar et al., 2002; Lockwood, 2002) and obesity (Nemmar et al., 2002; Sun el al., 2009; Kelishadi, Mirghaffari, Poursafa, \& Gidding, 2009) in rural areas relative to urban centers. Poorer health status in socioeconomically deprived and rural environments may reflect, in part, the inaccessibility of such built environmental features as public pools, recreation centers, physical fitness facilities, parks, sidewalks, and streetlights (Goldberg et al., 2000). Stewart et al (2011) conducted a study in the USA that explores potential county-level associations between diabetes prevalence among adult African Americans and five measures of the socioeconomic and built environment-persistent poverty, unemployment, rurality, number of fast food restaurants per capita, and number of convenience stores per capita. They found Diabetes prevalence rates in South Carolina are among the highest in the nation and there is association between the socioeconomic measures and diabetes (Stewart et al., 2011). 


\subsection{Environmental Pollution and Diabetes}

\subsubsection{Urban Pollution}

Diabetes is influenced not only by factors in each individual, but by the environment that surrounds the individual. There are many causes to Urban Contamination such as exposure to a chemical or other toxic substance originating from a source outside the building, e.g. motor vehicle exhaust fumes, construction activity, underground petrol spillage (Melius, Wallingford, Keelyside, \& Carpenter, 1984). One important regulated pollutant is particles equal or less than 10 microm in diameter (PM10), formed as a result of fossil-fuel combustion by motor vehicles and stationary sources such as power plants. PM10 are generated from combustion emissions such as automobile exhaust or wood or coal burning and industrial emissions from smelters, paper and steel mills, or cement plants. PM10 can deposit in the lower airways changing portions of the lung, even reaching the circulatory system and, therefore, are considered to be of greater health significance (Nemmar et al., 2002). Many researchers have found association between environmental pollution and the pathogenesis of diabetes (Papazafiropoulou, Kardara, \& Pappas, 2011) and especially with those considered as a sensitive population such as children and with cardiovascular diseases. Balfour and Kaplan (2002) reported that poor lighting, excessive noise, heavy traffic, and lack of public transit are associated with loss of physical function in adults over 55 years of age. They suggested that these detrimental environmental features discourage neighborhood excursions. In addition, individuals with diabetes are at greater risk of dying (Goldberg et al., 2001) and being hospitalized for heart disease during periods of high environmental pollution (Zanobetti \& Schwartz, 2002). This phenomenon has been explained partially due to the association between exposure to environmental pollution and markers of cardiovascular risk related to decreased heart rate variability (Creason et al., 2001; Tunnicliffe, Hilton, Harrison, \& Ayres, 2001) and increased levels of thrombotic and inflammatory factors (Donaldson, Stone, Seaton, \& MacNee, 2001; Peters et al., 2001) since inflammation is the key pathway leading to atherosclerosis and subsequent adverse cardiovascular events. The diabetic subjects with cardiovascular diseases are more susceptible to the detrimental effects of environmental pollution than diabetics without cardiovascular diseases (Goldberg et al., 2006; Filho et al., 2008). Other studies in children have showed that environmental pollution is linked with the development of T1DM (Hathout, Beeson, Ischander, Rao, \& Mace, 2006).

A lot of recent studies have showed a relationship between PM10 and diabetes mellitus (Lockwood 2002; Sun et al., 2009; Kelishadi, Mirghaffari, Poursafa, \& Gidding, 2009). The long-term exposure to environmental pollution particles, $2.5 \mathrm{~mm}$ in aerodynamic diameter (PM2.5) has been found to be associated with a higher relative risk of mortality among people with diabetes compared with the general population (Jerrett et al., 2005). Also similar association found between prevalence of T2DM and PM2.5 (Pearson, Bachireddy, Shyamprasad, Goldfine, \& Brownstein, 2010). A study by Brook et al., (Brook, Jerrett, Brook, Bard, \& Finkelstein, 2008) investigates the relation between diabetes and traffic-related $\mathrm{NO}^{2}$ in two different cities; Toronto and Hamilton. They found significant association between the diabetes prevalence and exposure to traffic-related $\mathrm{NO}^{2}$. Lee et al (Lee et al., 2006) have found similar association between the organic pollutants and prevalence of T2DM (see similar findings by Ukropec et al., 2010). Hathout et al. (2002) found association between Prevalence of T1DM and substances $\mathrm{O}^{3}, \mathrm{SO}^{2}$ and substances $\mathrm{NO}^{2}, \mathrm{SO}^{2}$ and $\mathrm{SO}^{4}$. This possible association between environmental pollution and increased incidence of diabetes should motivate policy makers to issue prevention policies to reduce air pollution and therefore, its, harmful consequences.

\subsubsection{Sick Building Syndrome}

Buildings are complex environments which can trap and concentrate pollutants as well as generate them. Outside pollutants find their way into buildings through air intakes and inadequate filtering systems. As long as ample ventilation ensures a constant supply of fresh air, indoor pollution problems may be kept to a minimum. However, general ventilation is often inadequate and office equipment may have no local exhaust system venting fumes to the outside (London Hazards Centre trust, 1990). Youle (1986) has found that air-conditioning systems giving rise to symptoms of sick building syndrome. Researchers have found association between the ventilation rates, $\mathrm{CO}^{2}$-concentrations with health problems in commercial and institutional (Seppänen, Fisk, \& Mendell, 1999) and Office Buildings (Apte, Fisk, \& Daisey, 2000). Sick building syndrome has a number of health symptoms such as: lethargy and tiredness, headache, dry blocked nose, sore dry eyes, sore throat, dry skin and/or skin rashes, allergy etc (World Health Organization, 1983; Morris \& Hawkins, 1987; Wilson \& Hedge, 1987). Allergic reaction in sensitive individuals was associated with chest tightness, difficulty in breathing, fever and headache. These health problems may aggravate diabetes. Certain materials are identified as causes of Contamination inside the buildings: exposure to chemical or other toxic agent generated within the office space, e.g. methyl alcohol from spirit duplicator, methacrylate from a copier, sulphur dioxide from a heating system, amines used in a humidification system, chlordane used as a pesticide (Melius et al., 1984). 


\subsection{Potential Solutions}

The green and sustainable buildings design aim is to design buildings that reduce the overall impact of the built environment on human health and the natural environment by: efficiently using energy, water, and other resources, protecting occupant health and improving employee productivity, and reducing waste, pollution and environmental degradation. These buildings theoretically use zero energy and do not release harmful substances to the environment, enrich the environment in a way or another thus it mimics the nature. Such buildings should provide healthy indoor environment to its inhabitants and expose them to adequate amount of natural light and ventilation, views of greenery, and close proximity to outdoor green space. Landscape architecture appears to be the primary key at the finest scale to sound mind and body and simply viewing nature reduces the stress of daily urban life (Ulrich, 1979; Jackson, 2011). Jackson and Kochtitzky (2001) advocate providing neighborhood opportunities for walking to accomplish routine activities such as shopping and going to work. Andersen et al., (1999) report that lifestyle activities such as structured aerobic exercise are effective in losing weight. Critical to this strategy is conducive neighborhood design. Physical activity is defined as the total of planned and repetitive movements of skeletal muscles, which are performed using energy. The beneficial effects of exercise in patients with T2DM have been recognized long time ago. Today, the beneficial role of exercise has been fully documented and exercise should be incorporated systematically in the treatment of patients with diabetes (Lecomte, Romon, Fosse, Simon, \& Fagot-Campagna, 2008; De Feo et al., 2006; Sato et al., 2007; La Monte et al., 2005). Moreover, exercise has a significant role in the regulation of blood glucose, improves insulin action, metabolism of proteins and fats, prevents complications of diabetes, improves muscle flexibility and strength, has beneficial effects on the cardiovascular system and increases life expectancy of the patients. In addition, physical activity is beneficial for the mental state of the individual, because it increases the energy of the human body, improves self-esteem and decreases depression (Lecomte et al., 2008; De Feo et al., 2006; Sato et al., 2007; Colberg, 2007). It is widely accepted that healthy nutrition is the basis for the treatment of T2DM. It contributes positively to the maintenance of blood glucose within normal range and minimizes the complications of the disease. Unfortunately the diet in urban dwellers are usually contains a greater proportion of refined carbohydrates, less fiber and more fat in comparison to that in rural environment (Stephen \& John, 1997).

Smart technology is defined as the technology used to make all electronic devices in a building act "smart" or more automated. The smart technology aim is to help people including those who have special needs i.e. disability to control the environment that is around them. Also, it helps them to get support and assistance from the surrounding environment. A smart home is a home that equipped with special structured wiring and devices that enable occupants to remotely control or program an array of automated home electronic devices by entering a single command (SearchSMB.com, 2007). Smart products and services can be divided into six categories (Roe, 2007) namely: comfort, energy management, multimedia, and entertainment, healthcare (European Senior Watch Observatory and Inventory, 2002), security, safety and communication. Kanstrup et al. (2008) reviewed the ICT designed for diabetics. They categorized it into the following categories: medical devices, self-management tools, education and Games, and websites and social networks. Recently, home tele-monitoring has been applied as a new approach in the management of diabetes mellitus, to generate a registry of diabetic patients and provide tools for patient tracking and follow-up, in which physiological and biological data are transferred from the patients' home to the tele-monitoring centre by giving physician access to monitor patient's information, enabling identification of at risk population interpret the data, and providing clinical decisions (Roine, Ohinmaa, \& Hailey, 2001). However, home tele-monitoring is an integral part of a broader view of deinstitutionalization and reflects a societal orientation toward maintaining patients in their homes (Canadian Home Care Association, 2008). Many studies concluded that home tele-monitoring is as effective in glycemic control as the traditional approach to home follow-up (Paré, Jaana, \& Sicotte, 2007).

\section{The Research Objectives and Methodology}

The research has a set of objectives and these are:

- To find out the relation between the frequency and progress of diabetes symptoms, the patient's lifestyle, and built environment settings

- To make recommendations on how to enhance the built environment to be healthy, supportive and assistive

To achieve the research objectives, a questionnaire survey was carried out to assess the patients' views about the potential impact of built environment on the progress of the disease. Necessary permission was obtained as the protocol was reviewed and approved by the medical ethical committee of the University of Dammam. The field survey was used to target 36 patients who visit diabetes' clinic, King Fahid Teaching Hospital of the University of Dammam, in March/April 2011. A questionnaire was prepared to inspect the diabetic patients' views about 
their environment and lifestyle. Patients were randomly chosen. The choice was based on the following criteria: the exclusion of severely ill, children, and women patients as interviews with women need special arrangements; and the inclusion of T1\& 2DM male adults, age 18-60 years old. During the appointment with each diabetic patient at diabetes' clinic, King Fahid Teaching Hospital of the University of Damamm, the patient was asked to fill in the questionnaire. Thirty three male patients filled in the questionnaire and three returned invalid questionnaires. The sample size however, (i.e. number of respondents) was small and the following simple statistical tests were applied: Mean, percentage, Chi-square for the test of significance, and Cramer's test of correlation. It should be mentioned that the study reports the significant results only (i.e. $0.5>$ P).

\section{The Field Survey Results}

The validity and reliability of the data was tested. The reliability (i.e. Cronbach Alpha) and validity values were 0.947 and 0.897. Patients are from Dammam and Al Khober cities and few are from AlAhsa city and Al Qateef city. The analysis of the questionnaire results showed that $21 \%$ of the respondents are between 20 to 40 years old, 55\% are between 41-60 years old and the rest are between 61-80 years old. Around one third of the respondents use insulin and tablets. Around $50 \%$ use tablets and less than a quarter use insulin. Around one third of the respondents have high last Fasting blood Glucose reading i.e. more than $200 \mathrm{mg} / \mathrm{dL}$. And one third has medium reading i.e. $151-200 \mathrm{mg} / \mathrm{dL}$ and the rest have low reading. Around $21 \%$ of the respondents said the Onset date of the disease is from one to five years, $42 \%$ said it is from $6-10$ years ago, $12 \%$ said that they have it from 11 to 15 years whereas $21 \%$ said they have more than 15 years ago. $91 \%$ of the respondents have lived in their present address more than 5 years and $6 \%$ have been less than 5 years whereas the rest did not reveal the period of residence. $24 \%$ have experienced accidents since the onset of the disease and $73 \%$ have not. $30 \%$ said that the highway is less than 500 meters from their homes, whereas $80 \%$ of the respondents said that the main road is less than 500 meters from their homes. $41 \%$ of the respondents pointed out that the public amenities are less than 200 meters and $44 \%$ said it is less than 500 meters. Around one third said the public gardens are less than 500 meters. However, $42 \%$ of the participants said it is more than 2000 meters from home. $41 \%$ said that the Recreation and sport Centre is more than 2000 meters from home, whereas around $22 \%$ said it is less than 500 meters from home.

More than half of the participants said that they have never or rarely done any morning exercises on daily basis (Table 1 and Figure 1). Only a quarter of the respondents have done frequently/ always morning exercises. More than one third of the surveyed patients said they never/ rarely walk for 30 minutes every day. Around the quarter said they did it frequently and only $10 \%$ said they always do it. Around $75 \%$ of the respondents said they often/ always watch TV or carry out office work on daily basis. $72 \%$ of the respondents said they often/ always eat fruits and vegetables every day. $27 \%$ of the respondents said they sometimes drink fizzy drinks and eat junk food every day. Around $20 \%$ said they smoke excessively on daily basis. Since they have lived in their homes, the respondents complained mostly from the following environmental issues: noise from the traffic, little sun penetration to their homes, noise from neighbors, difficulty to wonder around within the neighborhood, the lack of cleanness of the neighborhood (Table 2).

Table 1. The frequency of daily lifestyle activities of the patient since the onset of diabetes

\begin{tabular}{llllll}
\hline Daily lifestyle activity & Never & rarely & Sometimes & Often & Always \\
\hline Doing morning sport exercises & $36 \%$ & $18 \%$ & $21 \%$ & $9 \%$ & $15 \%$ \\
Walking for 30 minutes & $13 \%$ & $23 \%$ & $29 \%$ & $26 \%$ & $10 \%$ \\
TV watch or office work & $0 \%$ & $6 \%$ & $18 \%$ & $27 \%$ & $48 \%$ \\
Eating fruits and vegetables & $0 \%$ & $12 \%$ & $15 \%$ & $42 \%$ & $30 \%$ \\
Drinking fizzy drinks & $48 \%$ & $18 \%$ & $27 \%$ & $0 \%$ & $6 \%$ \\
Eating junk food meals & $39 \%$ & $33 \%$ & $27 \%$ & $0 \%$ & $0 \%$ \\
Excessive smoking & $78 \%$ & $0 \%$ & $3 \%$ & $6 \%$ & $13 \%$ \\
\hline
\end{tabular}

Sample size $=30$ 


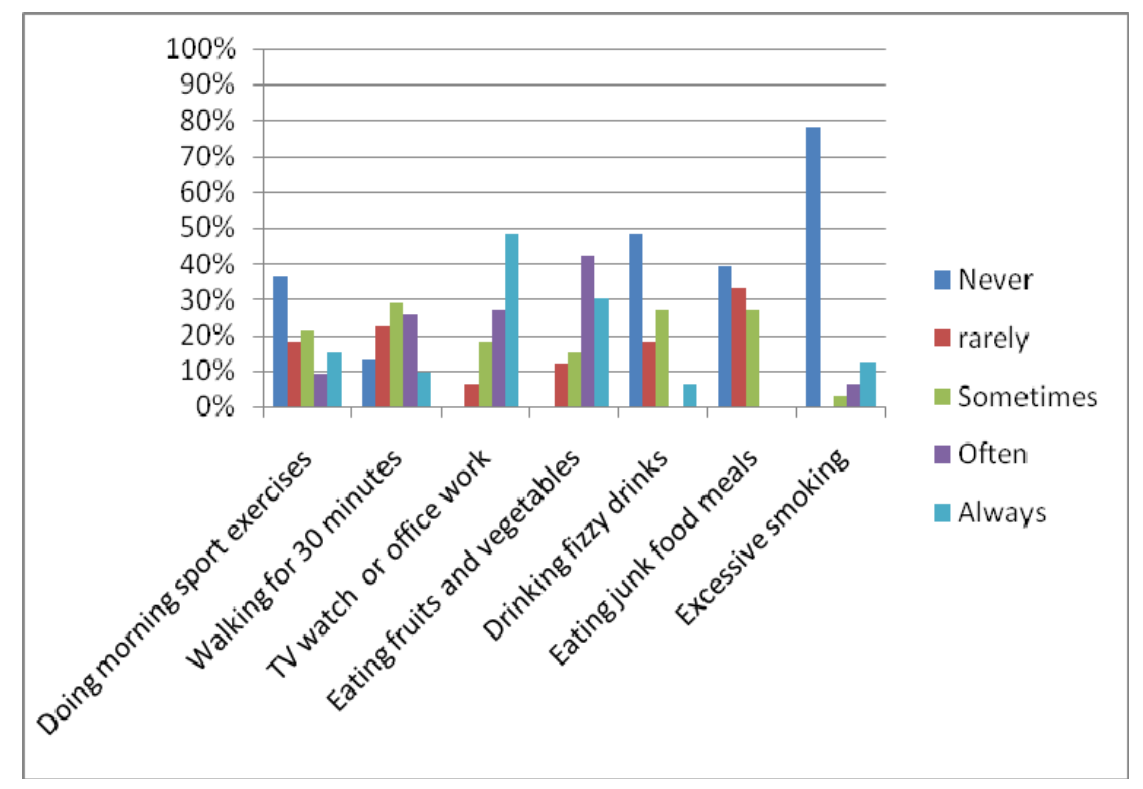

Figure 1. The frequency of daily lifestyle habits

Table 2. How often a number of conditions are experienced by the patient since he has resided in his/her present address

\begin{tabular}{llllll}
\hline Home and neighborhood environment's condition & Never & rarely & Sometimes & Often & Always \\
\hline Too little air/ ventilation & $70 \%$ & $3 \%$ & $18 \%$ & $6 \%$ & $3 \%$ \\
Annoying air draft & $69 \%$ & $9 \%$ & $13 \%$ & $3 \%$ & $6 \%$ \\
Hot air conditions & $58 \%$ & $18 \%$ & $18 \%$ & $3 \%$ & $3 \%$ \\
Little sun penetration & $55 \%$ & $6 \%$ & $15 \%$ & $18 \%$ & $6 \%$ \\
Poor air quality & $72 \%$ & $13 \%$ & $9 \%$ & $6 \%$ & $0 \%$ \\
Unpleasant outside views & $91 \%$ & $3 \%$ & $3 \%$ & $0 \%$ & $3 \%$ \\
Unpleasant odours & $58 \%$ & $15 \%$ & $24 \%$ & $0 \%$ & $3 \%$ \\
The lack of cleanness in the neighborhood & $52 \%$ & $12 \%$ & $30 \%$ & $6 \%$ & $0 \%$ \\
The quality of home finishing & $69 \%$ & $6 \%$ & $16 \%$ & $0 \%$ & $9 \%$ \\
The uncomfortable home organization and small size & $61 \%$ & $15 \%$ & $9 \%$ & $3 \%$ & $12 \%$ \\
The uncomfortable home furniture & $73 \%$ & $9 \%$ & $15 \%$ & $0 \%$ & $3 \%$ \\
Noise from the traffic & $39 \%$ & $10 \%$ & $23 \%$ & $16 \%$ & $13 \%$ \\
Noise from neighbors & $53 \%$ & $6 \%$ & $31 \%$ & $3 \%$ & $6 \%$ \\
Pollutant neighborhood & $61 \%$ & $16 \%$ & $23 \%$ & $0 \%$ & $0 \%$ \\
Difficulty to wonder around within the neighborhood & $56 \%$ & $9 \%$ & $22 \%$ & $3 \%$ & $9 \%$ \\
\hline
\end{tabular}

Sample size $=30$

The least complains were about the followings: the uncomfortable home furniture, poor air quality in the house, and unpleasant outside views (Table 2). Around one third said they always/ often suffer from the following health problems since they have had the diabetes: Paresthesia, blurred vision, extreme tiredness, and extra fat problems since the onset of the diseases (Table 3). $44 \%$ of the respondents said they suffer always/ often from blood pressure problems and 19\% experience drowsiness since they have the diabetes. However, around half of them said that they sometimes have stress and around one third have extreme tiredness. Few of the respondents said they experience loss of sensation particularly in the foot limbs or Cardiatric problems (Table 3). Only one third of the respondents use Remote $\mathrm{A} / \mathrm{C}$ and heating control and tele-services through the Internet and very few have remote lighting control (Table 4). Nearly all the respondents do not have: electronic medical devices and medical 
aids, remote lighting control, life safety System, virtual clinic/hospital tools, electronic Security and anti-burglary system and individual wellness monitoring tools.

Table 3. How often the patient has experienced a number of health problems since the onset of diabetes

\begin{tabular}{llllll}
\hline Type of symptoms and health problems & Never & rarely & Sometimes & Often & Always \\
\hline Paresthesia & $28 \%$ & $13 \%$ & $25 \%$ & $9 \%$ & $25 \%$ \\
Blurred vision & $27 \%$ & $18 \%$ & $18 \%$ & $18 \%$ & $18 \%$ \\
Extreme tiredness & $12 \%$ & $12 \%$ & $36 \%$ & $15 \%$ & $24 \%$ \\
Stress & $9 \%$ & $21 \%$ & $48 \%$ & $6 \%$ & $15 \%$ \\
Loss of sensation particularly in the foot limbs & $65 \%$ & $16 \%$ & $10 \%$ & $3 \%$ & $6 \%$ \\
Cardiatric problems & $75 \%$ & $9 \%$ & $6 \%$ & $0 \%$ & $9 \%$ \\
Blood pressure problems & $41 \%$ & $3 \%$ & $13 \%$ & $19 \%$ & $25 \%$ \\
Extra fat problems & $36 \%$ & $12 \%$ & $12 \%$ & $12 \%$ & $27 \%$ \\
Drowsiness & $34 \%$ & $19 \%$ & $28 \%$ & $13 \%$ & $6 \%$ \\
\hline Samp
\end{tabular}

Sample size $=30$

Table 4. How frequent the patient has used smart home systems

\begin{tabular}{lcccccc}
\hline Type of smart home systems & $\begin{array}{c}\text { Do not } \\
\text { have }\end{array}$ & $\begin{array}{c}\text { Never } \\
\text { used }\end{array}$ & $\begin{array}{c}\text { Rarely } \\
\text { used }\end{array}$ & $\begin{array}{c}\text { Sometimes } \\
\text { used }\end{array}$ & $\begin{array}{c}\text { Often } \\
\text { used }\end{array}$ & $\begin{array}{c}\text { Always } \\
\text { used }\end{array}$ \\
\hline Electronic medical Devices and medical aids & $100 \%$ & $0 \%$ & $0 \%$ & $0 \%$ & $0 \%$ & $0 \%$ \\
Remote A/C and heating control & $58 \%$ & $0 \%$ & $6 \%$ & $9 \%$ & $6 \%$ & $21 \%$ \\
Remote lighting control & $88 \%$ & $0 \%$ & $0 \%$ & $6 \%$ & $3 \%$ & $3 \%$ \\
Life safety System & $91 \%$ & $0 \%$ & $3 \%$ & $0 \%$ & $3 \%$ & $3 \%$ \\
Virtual clinic/hospital tools & $97 \%$ & $0 \%$ & $0 \%$ & $3 \%$ & $0 \%$ & $0 \%$ \\
Electronic Security and anti-burglary system & $94 \%$ & $3 \%$ & $3 \%$ & $0 \%$ & $0 \%$ & $0 \%$ \\
Individual wellness monitoring tools & $76 \%$ & $0 \%$ & $0 \%$ & $0 \%$ & $9 \%$ & $15 \%$ \\
Tele-services through the Internet & $36 \%$ & $0 \%$ & $21 \%$ & $12 \%$ & $9 \%$ & $21 \%$ \\
\hline
\end{tabular}

Sample size $=30$

In regards to cross tabulation results, only significant links between variables (i.e. $0.05>$ P) are reported here. The examination of data shows that the older patients drink less fizzy drinks than younger patients and this can be considered as a healthy phenomenon $(\mathrm{P}=0.011)$. The older patients seem to be more sensitive to the environmental conditions and complain more about the pollutant neighborhood than the younger patients $(\mathrm{P}=$ 0.020). Also, they suffer Extreme tiredness and loss of sensation particularly in the foot limbs more than younger patients $(\mathrm{P}=0.005)$. Patients with lower last fasting blood Glucose reading, walk more frequently than patients with higher Glucose reading $(\mathrm{P}=0.013)$. Al khober and Dammam's patients tend to walk more frequently than $\mathrm{AL}$ Ahsaa and Al Qateef patients $(\mathrm{P}=0.023)$. However, they watch more $\mathrm{TV}$ or carry out office work than $\mathrm{Al}$ Ahsaa and $\mathrm{Al}$ Qateef patients $(\mathrm{P}=0.033)$. Al Qateef patients drink more frequently fizzy drinks $(\mathrm{P}=0.003)$ and do excessive smoking more than other patients $(\mathrm{P}=0.026)$. However, as the sample of Al Qateef is small, the results cannot be generalized to the whole population. Al Qateef patients have suffered more frequently than other patients from the following sick indoor conditions: annoying air draft, hot air conditions, poor air quality, unpleasant odours and extreme tiredness $(\mathrm{P}=0.041, \mathrm{P}=0.0002, \mathrm{P}=0.00004, \mathrm{P}=0.023$ and $\mathrm{P}=0.039)$. Patients who tend to watch more frequently the TV or carry on office work are those who live in an area whereas the recreation and sport Centre is farther $(\mathrm{P}=0.015)$. Patients who experience more frequently the loss of sensation particularly in the foot limbs, said that they suffer more frequently from too little indoor air/ ventilation, annoying air draft and noise from neighbors $(\mathrm{P}=0.001, \mathrm{P}=0.0001$ and $\mathrm{P}=0.007)$. Patients, who experience more frequently from blood pressure problems, said they suffer more frequently from neighbors' noise $(\mathrm{P}=0.012)$ and 
those who experience more frequently from drowsiness said that they have higher difficulty in wandering around within the neighborhood $(\mathrm{P}=0.038)$.

\section{Discussion and Conclusion}

Results of the present study supported with the literature review showed the negative possible impact of the existing built environment conditions in the Eastern province, KSA on the diabetic patients. The survey Results showed that public gardens and recreation and sport Centers are not well located within the urban context. This would make it difficult for the patients to enjoy walking to and within these centers and parks particularly during the harsh hot weather that last around 6 month in KSA. Similar findings were found by Allanah, Ashley, \& Farley (2010) who stated that the ill designed urban settings would enforce people to adopt unhealthy lifestyle thus contribute to the development of T2DM. Therefore, it is no surprise to find that patients who tend to watch more frequently the TV or carry on office work are those who live in an area whereas the recreation and sport Centre is far from their homes. In respect to the lifestyle activities, more than half of the participants said that they have never or rarely done any morning exercises and more than one third said they never/ rarely walk for 30 minutes on daily basis. Around three quarters of the respondents often/ always watch TV or carry out office work every day meanwhile around the same percentages often/ always eat fruits and vegetables on daily basis. Around one third said they always/ often suffer a number of health problems. Eckel et al. (2004), Pekhan (1998) and Michaud et al (2001) have linked the passive entertainment, lack of physical activities and bad eating habits with the increasing incidence of the diabetes. The respondents complained mostly from a number of environmental issues such as noise from the traffic and neighbors, little sun penetration to their homes, difficulty to wonder around within the neighborhood, and the lack of cleanness of the neighborhood. The results showed that the frequency of the symptoms is higher for patients who suffer more frequently from indoor/ outdoor environmental/ urban conditions. The older patients seem to be more sensitive to the environmental conditions than the younger patients. Papazafiropoulou, Kardara, \& Pappas (2011); and Balfour and Kaplan (2002), found an association between the environmental pollution and the pathogenesis of diabetes, particularly among old people and these who have cardiovascular diseases (see also Goldberg et al., 2006; Filho et al., 2008). Patients who experience more frequently from drowsiness said that they have higher difficulty in wondering around within the neighborhood. This clearly indicates that the present built environment in the Eastern province, KSA is not designed to be friendly and assistive to users. Previous research has generally points out that poorly designed urban settings would discourage neighborhood excursions (see for instance Goldberg et al., 2000)

The previous research demonstrated the usefulness of smart technology for diabetic patients (see for instance Roine, Ohinmaa, \& Hailey, 2001), though this research found that it is hardly used by the participating patient. The researchers would like to point out the importance of providing healthy built environment to citizens. However, this built environment whether it is indoor or outdoor should be supported and equipped with smart tools that assist diabetic patients in their daily life. The researchers appreciate the limitation of the research but argue that it can be used as a foundation for further research. Future research should explore how a healthy environment should be designed and implemented taking into account the local environmental, cultural and lifestyle issues in KSA and Eastern Province in particular. It also may explore the impact of implementing smart tools on Urban and home levels on diabetic patients' life and interaction with the built environment.

\section{References}

Alberti, K. G., \& Zimmet, P. Z. (1998). Definition, diagnosis and classification of diabetes mellitus and its complications. Part 1: diagnosis and classification of diabetes mellitus provisional report of a WHO consultation. Diabet Med., 15, 539-553. http://dx.doi.org/10.1002/(SICI)1096-9136(199807)15:7<539::AID -DIA668 $>3.0 . \mathrm{CO} ; 2-\mathrm{S}$

Allanah, L., Ashley, K., \& Farley, E. (2010). Diabetes and the built environment: Contributions from an emerging interdisciplinary research programme.

American Diabetes Association. (2006). Standards of medical care in diabetes-Diab. Care, 29(Suppl. 1), S4-S42.

Al-Nozha, M. M., Al-Maatouq, M. A., \& Al-Mazrou, Y. Y. (2004). Diabetes mellitus in Saudi Arabia. Saudi Med. J., 25(11), 1603-10.

Andersen, R. E., Wadden, T. A., Bartlett, S. J., Zemel, B., Verde, T. J., \& Franckowiak, S. C. (1999). Effects of lifestyle activity versus structured aerobic exercise in obese women: a randomized trial. J. Am. Med. Assoc., 281(4), 335-340. http://dx.doi.org/10.1001/jama.281.4.335

Apte, M. G., Fisk, W. J., \& Daisey, J. M. (2000). Associations Between Indoor CO2 Concentrations and Sick Building Syndrome Symptoms in US Office Buildings. Proceedings of Healthy Buildings, 1, 133-138. 
Atkins, R. (2005). The epidemiology of chronic kidney disease. Review, Kidney Int. Suppl., 94(Apr), S14-S18. http://dx.doi.org/10.1111/j.1523-1755.2005.09403.x

Alzaid, A. (1997). Time to declare war on diabetes. Annals of Saudi Medicine, 17, 154-155.

Balfour, J. L., \& Kaplan, G.A. (2002). Neighbourhood environment and loss of physical function in older adults: evidence from the Alameda county study. Am. J. Epidemiol., 155(6), 507-515. http://dx.doi.org/10.1093/aje/155.6.507

Bray, R., Vakil, C., \& Elliott, D. (2005). Report on public health and urban sprawl in Ontario: a review of the pertinent literature. Environmental health committee, Ontario College of Family Physicians. Retrieved from http://www.ocfp.on.ca/docs/publications/urbansprawl.pdf

Brook, R. D., Jerrett, M., Brook, J. R., Bard, R. L., \& Finkelstein, M. M. (2008). The relationship between diabetes mellitus and traffic-related air pollution. J. Occup. Environ. Med., 50, 32-8. http://dx.doi.org/10.1097/JOM.0b013e31815dba70

Canadian Home Care Association. (2008). Website Integration through information communication technology for home care in Canada. Retrieved from http://www.cdnhomecare.ca/media.php?mid=1840

Chaturvedi, N, Stevens, L. K., Fuller, J. H., Lee, E. T., Lu, M. (2001). Risk factors, ethnic differences and mortality associated with lower-extremity gangrene and amputation in diabetes: The WHO Multinational Study of Vascular Disease in Diabetes. Diabetologia, 44, 65-71. http://dx.doi.org/10.1007/PL00002941

Colberg, S. R. (2007). Physical activity, insulin action, and diabetes prevention and control. Curr. Diabetes Rev. 3(3), 176-84. http://dx.doi.org/10.2174/157339907781368986

Creason J., Neas, L., Walsh, D., Williams, R., Sheldon, L., \& Liao, D. (2001). Particulate matter and heart rate variability among elderly retirees: the Baltimore 1998 PM study. J. Exp. Anal. Environ. Epidemiol., 11, 116-22. http://dx.doi.org/10.1038/sj.jea.7500154

Cubbin, C., Sundquist, K., Ahlen, H., Johnansson, S. E., Winkleby, M. A., \& Sundquist, J. (2006). Neighbourhood deprivation and cardiovascular disease risk factors: protective and harmful effects. Scand J. Public Health, 34, 228-237. http://dx.doi.org/10.1080/14034940500327935

De Feo, P., Di Loreto, C., Ranchelli, A., Fatone, C., Gambelunghe, G., \& Lucidi, P. (2006). Exercise and diabetes. Acta Biomed., 77(Suppl 1), 14-7.

Diex Roux, A. V., Merkin, S. S., Arnett, D., Chambless, L., Massing, A., Nieto, J., Sorlie, P., Szklo, M., Tyroler, H. A., \& Watson, R. L. (2001). Neighborhood of residence and incidence of coronary heart disease. N. Engl. J. Med., 345(2), 99-106. http://dx.doi.org/10.1056/NEJM200107123450205

Donaldson, K., Stone V., Seaton A., \& MacNee W. (2001). Ambient particle inhalation and the cardiovascular system: Potential mechanisms. Environ Health Perspect, Suppl 7, 109.

Ebbeling, C. B., Pawlak, D. B., \& Ludwig, D. S. (2002). Childhood obesity: public-health crisis, common sense cure. The Lancet, 360(9331), 473-482. http://dx.doi.org/10.1016/S0140-6736(02)09678-2

Eckel, R. H., York, D. A., Rössner, S., Hubbard, Van C., Ian, Jeor, Sachiko T. St, Hayman, L. L., Mullis, R. M., \& Blair, S. N. (2004). Obesity, a Worldwide Epidemic Related to Heart Disease and Stroke: Executive Summary. AHA Conference Proceedings Prevention Conference VII. Circulation, 110, 2968-2975.

European Senior Watch Observatory and Inventory. (2007). Older People and Information Society. (2002). Retrieved from http:// www.seniorwatch.de/reports/SW_D32_Final.pdf

Filho, P. M. A., Pereira, L. A. , Arbex, F. F., Arbex, M., Conceição, G. M., \& Santos, U. P. (2008). Effect of air pollution on diabetes and cardiovascular diseases in São Paulo, Brazil. Braz. J. Med. Biol. Res., 41, 526-32.

Goldberg, M. S., Burnett, R. T., Yale, J. F., Valois. M. F., Brook, J. R. (2006). Associations between ambient air pollution and daily mortality among persons with diabetes and cardiovascular disease. Environ Res., 100, 255-67. http://dx.doi.org/10.1016/j.envres.2005.04.007

Goldberg, M. S., Burnett, R. T., Bailar, JC 3rd, Brook, J., Bonvalot, Y., \& Tamblyn, R. (2001). The association between daily mortality and ambient air particle pollution in Montreal, Quebec. 2. Cause specific mortality. Environ. Res., 86, 26-36. http://dx.doi.org/10.1016/j.envres.2005.04.007

Goldberg, M. S., Bailar, III J. C., Burnett, R. T., Brook. J. R., Tamblyn. R., \& Bonvalot, Y. (2000). Identifying subgroups of the general population that may be susceptible to short-term increases in particulate air pollution: A time series study in Montreal, Quebec. Res. Rep. Health Effects Inst., 97, 7-113. 
Hathout, E. H., Beeson, W. L., Ischander, M., Rao, R., \& Mace, J. W. (2006). Air pollution and type 1 diabetes in children. Pediatr Diabetes, 7, 81-7. http://dx.doi.org/10.1111/j.1399-543X.2006.00150.x

Hathout, E. H., Beeson, W .L., Nahab, F., Rabadi, A., Thomas, W., \& Mace J. W. (2002). Role of exposure to air pollutants in the development of type 1 diabetes before and after $5 \mathrm{yr}$ of age. Pediatr Diabetes, 3, 184-8. http://dx.doi.org/10.1034/j.1399-5448.2002.30403.x

International Diabetes Federation. (2007). Diabetes Atlas, International Diabetes Federation, Brussels. http://da3.diabetesatlas.org/index $74 \mathrm{cc.html}$

International Diabetes Federation. (2009). Diabetes Atlas, 4th ed., International Diabetes Federation, Brussels. http://www.diabetesatlas.org/

Jackson, R. J., \& Kochtitzky, C. (2001). Creating a Healthy Environment: The Impact of the Built Environment on Public Health. Sprawl Watch Clearinghouse, Washington, DC.

Jackson, R. J. (2011). What Olmstead [sic] Knew. Western City. Retrieved from http://www.westerncity.com/Mar01Olmstead.htm

Jeppesen, P., \& Bek, T. (2004). The occurrence and causes of registered blindness in diabetes patients in Arhus $\begin{array}{llll}\text { County, Denmark. Acta Ophthalmol. } & \text { Scand., }\end{array}$ http://dx.doi.org/10.1111/j.1600-0420.2004.00313.x

Jerrett, M., Burnett, R. T., Ma, R., Pope, C. A. 3rd, Krewski, D., \& Newbold, K. B. (2005). Spatial analysis of air pollution and mortality in Los Angeles. Epidemiology, 16, 727-36. http://dx.doi.org/10.1097/01.ede.0000181630.15826.7d

Kanstrup, Anne Marie, Bertelsen, Pernille, Glasemann, Marie, \& Boye, Niels. (2008). Design for More: an Ambient Perspective on Diabetes. Retrieved from http://portal.acm.org/citation.cfm?doid=1795234.1795252

Kelishadi, R., Mirghaffari, N., Poursafa, P., \& Gidding, S. S. (2009). Lifestyle and environmental factors associated with inflammation, oxidative stress and insulin resistance in children. Atherosclerosis, 203, 311-9. http://dx.doi.org/10.1016/j.atherosclerosis.2008.06.022

Krishnan, S., Cozier, Y. C., Rosenberg, L., \& Palmer, J. R. (2010). Socioeconomic status and incidence of type 2 diabetes: results from the Black Women's Health Study. Am. J. Epidemiol., 171, 564-570. http://dx.doi.org/10.1093/aje/kwp443

La Monte, M., Blair, St., \& Church, T. (2005). Physical activity and diabetes prevention. J. Appl. Physiol., 99, 1205-1213. http://dx.doi.org/10.1152/japplphysiol.00193.2005

Lee, D. H., Lee, I. K., Song, K., Steffes, M., Toscano, W., \& Baker, B. A. (2006). A strong dose response relation between serum concentrations of persistent organic pollutants and diabetes: Results from the National Health and Examination Survey 1999-2002. Diabetes Care, 29, 1638-44. http://dx.doi.org/10.2337/dc06-0543

Lecomte, P., Romon, I., Fosse, S., Simon, D., \& Fagot-Campagna, A. (2008). Self-monitoring of blood glucose in people with type 1 and type 2 diabetes living in France: the Entred study 2001. Diabetes Metab., 34(3), 219-26. http://dx.doi.org/10.1016/j.diabet.2007.11.005

Lockwood, A. H. (2002). Diabetes and air pollution. Diabetes Care, 25, 1487-8. http://dx.doi.org/10.2337/diacare.25.8.1487

London Hazards Centre trust. (1990). Sick building syndrome: Causes, Effects and Control. London Hazards Centre.

Madhu, K. \& Sridhar. G. R. (2001) Coping with diabetes: A paradigm for coping with chronic illness. International Journal of Diabetes in Developing Countries, 21, 103-111.

Mayfield-Smith, Kathy. (2011). Diabetes and the socioeconomic and built environment: geovisualization of disease prevalence and potential contextual associations using ring maps. International Journal of Health Geographics, 10, 18. http://dx.doi.org/10.1186/1476-072X-10-18

Melius, J., Wallingford, K., Keelyside, R., \& Carpenter, J. (1984). Indoor air quality-the NIOSH experience. Ann. Am. Conf. Gov. Ind. Hyg., 10, 3-7. 
Michaud, J. L., Boucher, F., Melnyk, A., Gauthier, F., Goshu, E., Lévy, E., ... Fan, Chen-Ming. (2001). Sim1 haploinsufficiency causes hyperphagia, obesity and reduction of the paraventricular nucleus of the hypothalamus. Human Molecular Genetics, 10(14), 1465-1473. http://dx.doi.org/10.1093/hmg/10.14.1465

Morris, L. \& Hawkins, L. (1987). The role of stress in the sick building syndrome. In B. Siefert, H. Esdon, M. Fischer, H. Ruden and J. Wegner (eds.), Indoor Air '87, Proceedings of the 4th International Conference on Indoor Air Quality and Climate, Berlin (West): Institute for Water, Soil and Air Hygiene, Vol. 2, pp. 566-571.

Nemmar, A., Hoet, P. H., Vanquickenborne, B., Dinsdale, D., Thomeer, M., Hoylaerts, M. F. (2002). Passage of inhaled particles into the blood circulation in humans. Circulation, 105, 411-4. http://dx.doi.org/10.1161/hc0402.104118

Papazafiropoulou, Athanasia K., Kardara, Marina S., \& Pappas, Stavros I. (2011). Environmental Pollution and $\begin{array}{lllll}\text { Diabetes Mellitus. Recent Patents on } & \text { Biomarkers, }\end{array}$ http://dx.doi.org/10.2174/2210310411101010044

Paré, Guy, Jaana, Mirou, \& Sicotte, Claude. (2007). Systematic review of home telemonitoring for chronic diseases: the evidence base. J. Am. Med. Inform. Assoc., 14(3), 269-77. http://dx.doi.org/10.1197/jamia.M2270

Pearson, J. F., Bachireddy, C., Shyamprasad, S., Goldfine, A. B., \& Brownstein J. S. (2010). Association between fine particulate matter and diabetes prevalence in the US. Diabetes Care, 33, 2196-201. http://dx.doi.org/10.2337/dc10-0698

Peckhan, C. (1998). Fetal and child development. In Marinker M, Peckham M (eds). Clinical futures. Chapter 7; BMJ Books.

Peters, A., Frohlich, M., Doring, A., Immervoll, T., Wichmann, H. E., \& Hutchinson, W. L. (2001). Particulate air pollution is associated with an acute phase response in men; results from the MONICA-Augsburg Study. Eur. Heart J., 22, 1198-204. http://dx.doi.org/10.1053/euhj.2000.2483

Roe, Patrick R.W.(ed). (2007). Towards an inclusive future Impact and wider potential of information and communication technology. Edited by Swiss Federal Institute of Technology of Lausanne. Retrieved from http://www.tiresias.org/cost219ter/inclusive_future/inclusive_future_book.pdf

Roine, R., Ohinmaa, A., \& Hailey, D. (2001). Assessing telemedicine: a systematic review of the literature. CMAJ, 165(6), 765-71. Retrieved from http://www.pubmedcentral.nih.gov/articlerender.fcgi?tool=pubmed \&pubmedid $=11584564$

Schulze, M. B., Manson, J. E., Willett, W. C., \& Hu, F. B. (2003). Processed meat intake and incidence of Type 2 diabetes in younger and middle-aged women. Diabetologia, 46(11), 1465-1473. http://dx.doi.org/10.1007/s00125-003-1220-7

Seppänen, O. A., Fisk, W. J., \& Mendell, M. J. (1999). Association of ventilation rates and CO2-concentrations with health and other responses in commercial and institutional buildings. Indoor Air, 9, 226-252. http://dx.doi.org/10.1111/j.1600-0668.1999.00003.x

Sato, Y., Nagasaki, M., Kubota, M., Uno, T., \& Nakai, N. (2007). Clinical aspects of physical exercise for diabetes/metabolic syndrome. Diabetes Res. Clin. Pract., 77(Suppl 1), $\quad$ S87-91. http://dx.doi.org/10.1016/j.diabres.2007.01.039

SearchSMB.com Definitions (Powered by WhatIs.com). (2007). Smart technology. Retrieved from http://searchsmb.techtarget.com/sDefinition/0,,sid44_gci540859,00.html

Stephen, O. \& John, S. (1997). Clinical review, Science medicine and the future non-insulin dependent diabetes mellitus the gathering stom. BMJ, 14, 955.

Stewart, J. E., Battersby, S. E., Lopez-De, F. A., Remington, K. C., Hardin, J. W., Sun, Q., ... Mikolaj, M. B. (2009). Ambient air pollution exaggerates adipose inflammation and insulin resistance in a mouse model of diet-induced obesity. Circulation, 119, 538-46. http://dx.doi.org/10.1161/CIRCULATIONAHA.108.799015

Tunnicliffe, W. S., Hilton, M., Harrison, R. M., \& Ayres, J. G. (2001). The effect of sulphur dioxide exposure on indices of heart rate variability in normal and asthmatic adults. Eur. Respir. J., 17, 604-8. http://dx.doi.org/10.1183/09031936.01.17406040 
Ukropec, J., Radikova, Z., Huckova, M., Koska, J., Kocan, A., Sebokova, E. (2010). High prevalence of prediabetes and diabetes in a population exposed to high levels of an organochlorine cocktail. Diabetologia, 53, 899-906. http://dx.doi.org/10.1007/s00125-010-1683-2

Ulrich, R. S. (1979). Visual landscapes and psychological well-being. Landsc. Res., 4, 17-23. http://dx.doi.org/10.1080/01426397908705892

Wilson, S., \& Hedge, A. (1987). The Office Environment Survey: A Study of Building Sickness, Building Use Studies Ltd., London, U.K.

World Health Organization. (1983). Indoor air pollutants: exposure and health effects. EURO Reports and Studies 78, World Health Organization.

Youle, A. (1986). Occupational hygiene problems in office environments: the influence of building services. Ann Occ Hyg, 30(3), 275-287.

Zanobetti, A., \& Schwartz J. (2002). Cardiovascular damage by airborne particles: Are diabetics more susceptible? Epidemiology, 13, 588-92. http://dx.doi.org/10.1097/00001648-200209000-00016

\section{Appendix}

\begin{tabular}{|c|c|c|c|}
\hline Reference & Sample type & Sample size & Place \\
\hline $\begin{array}{l}\text { Al-Nozha, Al-Maatouq, \& } \\
\text { Al-Mazrou, } 2004\end{array}$ & $\begin{array}{l}\text { Saudi subjects in the age group of } \\
30-70 \text {-years of selected } \\
\text { households over a } 5 \text {-year period } \\
\text { between } 1995 \text { and } 2000\end{array}$ & 16917 participants & KSA \\
\hline Atkins, 2005 & Adults & $\mathrm{N}=11247$ & Victoria, Australia \\
\hline Allanah, Ashley, \& Farley, 2010 & Review of previous studies & - & Canada \\
\hline Balfour and Kaplan, 2002 & $\begin{array}{l}\text { participants in the Alameda } \\
\text { County Study who were aged } 55 \\
\text { years and older and functionally } \\
\text { healthy }\end{array}$ & 883 & $\begin{array}{l}\text { Alameda County, } \\
\text { USA }\end{array}$ \\
\hline Bray, Vakil, \& Elliott, 2005 & Literature review & - & Canada \\
\hline $\begin{array}{l}\text { Chaturvedi, Stevens, Fuller, Lee, } \\
\& \text { Lu, } 2001\end{array}$ & $\begin{array}{l}\text { Men and women aged } 35 \text { to } 55 \\
\text { years }\end{array}$ & $\mathrm{N}=3443$ & $\begin{array}{l}\text { American Indian, } \\
\text { Cuban, European } \\
\text { and East Asian } \\
\text { WHO centers }\end{array}$ \\
\hline Creason et al., 2001 & $\begin{array}{l}\text { Elderly (mean age } 82 \text { ) } \\
\text { nonsmoking residents }\end{array}$ & $\mathrm{N}=56$ & $\begin{array}{l}\text { Retirement center in } \\
\text { Baltimore County, } \\
\text { Maryland, USA }\end{array}$ \\
\hline Cubbin et al., 2006 & $\begin{array}{l}\text { Women and men aged over } 20 \\
\text { living in } 434 \text { neighborhoods }\end{array}$ & $\mathrm{N}=5883$ & Taiwan \\
\hline De Feo et al., 2006 & Literature review & & \\
\hline Diex Roux et al., 2001 & Low and high income persons & $\begin{array}{l}\mathrm{N}=13009 \\
\text { participants }\end{array}$ & USA \\
\hline $\begin{array}{l}\text { Donaldson, Stone, Seaton, \& } \\
\text { MacNee, } 2001\end{array}$ & Elderly individuals & - & UK \\
\hline Eckel et al., 2004 & Executive Summary & Varied & varied \\
\hline Filho et al., 2008 & $\begin{array}{l}\text { diabetic and non-diabetic patients } \\
\text { who have cardiovascular diseases }\end{array}$ & $\begin{array}{l}\mathrm{N}=45000 \\
\text { cardiovascular } \\
\text { emergency room } \\
\text { visits }\end{array}$ & São Paulo, Brazil \\
\hline Goldberg et al., 2000 & residents of Montreal & $\mathrm{N}=140939$ & Montreal, Quebec \\
\hline
\end{tabular}


Goldberg et al., 2001

Goldberg et al., 2006

Hathout et al, 2002

Hathout, Beeson, Ischander, Rao,

\& Mace, 2006

Jerrett et al., 2005

Jeppesen, P. \& Bek, T., 2004

Kelishadi, Mirghaffari, Poursafa, \& Gidding, 2009

Krishnan, Cozier, Rosenberg, \& Palmer, 2010

Lee et al., 2006

Lecomte, Romon, Fosse, Simon, \& Fagot-Campagna, 2008

Nemmar et al.; 2002

Michaud et al., 2001

Papazafiropoulou, Kardara, \& and Pappas, 2011

Peters et al., 2001

Seppänen, Fisk, \& Mendell, 1999

Stewart et al., 2011

Sun el al., 2009

Tunnicliffe, Hilton, Harrison, \& Ayres, 2001

Ukropec et al., 2010

Zanobetti, \& Schwartz, 2002 residents of Montreal

residents of Montreal

healthy children before and after 5 year of age

Children

Respiratory clinic patients

Diabetes patients

children, aged $10-18$ years

African-American women, aged 30-69 years,

Adult participants

Healthy volunteers

Experimentation on Mice

Literature review

Randomly selected men aged 45 to 64 years free of cardiovascular disease

Mixed

Adults aged 18 and older enrolled in South Carolina Medicaid, between July 2008 and June 2009

Male C57BL/6 mice

Normal and 12 asthmatic adults

Adults

Medicare recipients (aged_65 years) residing in Allegheny

County (Pittsburgh area), Pennsylvania

\begin{tabular}{|c|c|}
\hline $\mathrm{N}=140939$ & $\begin{array}{l}\text { Montreal, Quebec, } \\
\text { Canada }\end{array}$ \\
\hline $\begin{array}{l}\mathrm{N}=-(\text { Time series } \\
\text { study) }\end{array}$ & $\begin{array}{l}\text { Montreal, Quebec, } \\
\text { Canada }\end{array}$ \\
\hline $\mathrm{N}=3$ & USA \\
\hline $\mathrm{N}=402$ & California, USA \\
\hline $\mathrm{N}=2360$ & Los Angeles, USA \\
\hline $\mathrm{N}=7527$ & $\begin{array}{l}\text { Århus County, } \\
\text { Denmark }\end{array}$ \\
\hline $\mathrm{N}=374$ & IRAN \\
\hline 46382 participants & USA \\
\hline $\mathrm{N}=2016$ & Korea \\
\hline $\mathrm{N}=5$ & Belgium \\
\hline $\begin{array}{l}\text { The number of Mice } \\
\text { is: } 15 \operatorname{Sim} 1^{+/-} \text {males, } \\
13 \operatorname{Sim} 1^{+/+} \text {males, } 19 \\
\operatorname{Sim} 1^{+/-} \text {females and } \\
17 \operatorname{Sim} 1^{+/+} \text {females. }\end{array}$ & Canada \\
\hline $\mathrm{N}=631$ & Germany \\
\hline $\mathrm{N}=30000$ subjects & Helsinki, Finland \\
\hline $\mathrm{N}=442830$ & USA \\
\hline $\mathrm{n}=14$ per group & USA \\
\hline $\mathrm{N}=12$ & UK \\
\hline $\mathrm{N}=2047$ & $\begin{array}{l}\text { Bratislava, Slovak } \\
\text { Republic }\end{array}$ \\
\hline $\mathrm{N}=55019$ & USA \\
\hline
\end{tabular}

